\title{
INFRARED SPECTROSCOPY OF NAPHTHALENE AGGREGATION AND CLUSTER FORMATION IN ARGON MATRICES
}

\author{
J. E. Roser ${ }^{1,2}$ and L. J. Allamandola ${ }^{3}$ \\ ${ }^{1}$ NASA Ames Research Center, Mail Stop 245-6, Bldg. N245, Room 148, P.O. Box 1, Moffett Field, CA 94035, USA; Joseph.E.Roser@nasa.gov \\ ${ }^{2}$ SETI Institute, 189 Bernardo Ave., Mountain View, CA 94043, USA \\ ${ }^{3}$ NASA Ames Research Center, Moffett Field, CA 94035, USA \\ Received 2010 February 25; accepted 2010 August 27; published 2010 October 5
}

\begin{abstract}
Fourier-transform, mid-infrared absorption spectra of mixed argon/naphthalene matrices at $5 \mathrm{~K}$ are shown with ratios of argon-to-naphthalene that vary from 1000 to 0 . These spectra show the changes as naphthalene clustering and aggregation occurs, with moderate spectral shifts affecting the $\mathrm{C}-\mathrm{H}$ vibrational modes and relatively small or no shifts to the $\mathrm{C}-\mathrm{C}$ and $\mathrm{C}-\mathrm{C}-\mathrm{C}$ vibrational modes. The possible contribution of homogeneous naphthalene clusters to the interstellar unidentified infrared bands is discussed. The contribution of polycyclic aromatic hydrocarbon (PAH) clusters to the $7.7 \mu \mathrm{m}$ emission plateau and the blue shading of the $12.7 \mu \mathrm{m}$ emission band are identified as promising candidates for future research. In addition, since PAH clusters are model components of Jupiter and Titan's atmospheres, the information presented here may also be applicable to the spectroscopy of these objects.
\end{abstract}

Key words: astrochemistry - infrared: ISM - ISM: lines and bands - ISM: molecules - molecular data

Online-only material: color figure

\section{INTRODUCTION}

A common phenomenon associated with the interstellar medium is the emission of unidentified infrared bands (or UIBs) at 3.3, 6.2, 7.7, 8.6, 11.2, and $16.4 \mu \mathrm{m}(3030,1610$, $1300,1160,890$, and $610 \mathrm{~cm}^{-1}$, respectively). The emitters of these bands are generally associated with gas-phase aromatic molecules, to date, primarily neutral and ionized polycyclic aromatic hydrocarbons or PAHs (see, for example, Peeters et al. 2004; Tielens 2008; Ricks et al. 2009; Boersma et al. 2010, and references therein) and possibly protonated PAHs (Ricks et al. 2009; Knorke et al. 2009) and aromatic heterocycles (Hudgins et al. 2005; Mattioda et al. 2003, 2005, 2008). Efforts to synthesize the UIBs typically calculate emission spectra under interstellar conditions from a large database of experimentally measured or theoretically computed infrared absorption measurements of aromatic molecules (Peeters et al. 2004; Mulas et al. 2006; Bauschlicher et al. 2008, 2009). The match between synthesized spectra and observations is close enough that the UIBs are now commonly referred to as the PAH bands or Aromatic Infrared Bands (or AIBs).

The AIBs overlap a series of wider emission "plateaus" which vary independently from the emission bands (Bregman et al. 1989; Boersma et al. 2009; Tielens 2008) and which are attributed to clusters of PAH molecules (Peeters et al. 2006; Kimura et al. 2007). The identity of the plateau clusters is significantly less well known due to the relative lack of experimental data for PAH clusters with respect to individual PAHs (see the discussion in Rapacioli et al. 2006 for example), the problem of accurately accounting for dispersive interactions in quantum chemical models of PAH clusters (Tsuzuki et al. 2004; Saeki et al. 2006; Rapacioli et al. 2009), and the problem of determining accurate nucleation and evaporation rates for PAH clusters in interstellar environments (Rapacioli et al. 2006). In the case of PAH clusters, this lack of data is particularly acute since these clusters are important both as a potential emitter of the emission plateaus and as a source of individual PAHs through evaporation of their sub-units (Rapacioli et al. 2006; Joblin et al. 2009).
Here we report an experimental study on naphthalene clusters to start to provide this information. This paper is a first step toward addressing the infrared properties of naphthalene clusters through transmission measurements of the infrared absorption spectra of naphthalene molecules in various degrees of aggregation within an argon ice matrix. Naphthalene is, of course, a very small PAH by interstellar standards. However, this makes it particularly attractive from a theoretical point of view since it provides empirical data on a system which is small enough to make it computationally tractable. In the absence of a complete theory of the nucleation of PAH clusters, it is not yet clear how the naphthalene cluster structures in solid solutions relate to those in molecular beam experiments and how either of these relate to structures of interstellar naphthalene clusters. One motivation for this paper is to provide empirical data useful for progress in this direction.

Naphthalene is also distinguished for study by recent observational results suggesting that some interstellar environments have sufficiently attenuated UV radiation fields that permit the survival of small PAHs. In particular, Iglesias-Groth et al. (2008) claimed detection of the naphthalene cation in the Perseus molecular cloud complex while Vijh et al. (2005) attribute the blue luminescence phenomenon in the Red Rectangle to three- and four-ringed neutral PAH molecules. It seems reasonable that a two-ringed PAH such as naphthalene might also be present in a region where three- and four-ringed PAHs are indicated.

Homogeneous naphthalene clusters have been well studied using multiphoton ionization spectroscopy (Wessel \& Syage 1990; Benharash et al. 1999; Fujiwara \& Lim 2003; Saigusa \& Lim 1995; Gilliéron et al. 2007), mass-selective ionizationloss stimulation Raman spectroscopy (Schaeffer et al. 1995; Benharash et al. 1999; Kim et al. 1999), and photoelectron spectroscopy (Ando et al. 2008) but systematic studies of their infrared absorption in the AIB range have yet to be conducted. The structures of neutral naphthalene clusters as large as the tetramer have been determined for molecular beam experiments (see Gilliéron et al. 2007 for a recent discussion, and references therein for more detail). 
Table 1

Summary of Molecular Data for the Spectra Shown in Figures 1-3

\begin{tabular}{lcccccc}
\hline \hline \multicolumn{1}{c}{ Ar/Nap Ratio } & $1000 \pm 300$ & $530 \pm 90$ & $200 \pm 50$ & $160 \pm 40$ & $47 \pm 7$ & $6 \pm 2$ \\
\hline Argon thickness $(\mu \mathrm{m})$ & $6.1 \pm 1.5$ & $10.0 \pm 0.8$ & $3.3 \pm 0.5$ & $7.3 \pm 1.7$ & $2.1 \pm 0.3$ & $2.2 \pm 0.8$ \\
Naphthalene density $^{\mathrm{a}}$ & $2.6 \pm 0.3$ & $8.4 \pm 1.2$ & $7.2 \pm 1.4$ & $20 \pm 2$ & $20 \pm 1$ & $160 \pm 20$ \\
Monomer fraction $^{\mathrm{b}}$ & $0.90 \pm 0.03$ & $0.82 \pm 0.03$ & $0.59 \pm 0.08$ & $0.51 \pm 0.09$ & $0.10 \pm 0.04$ & $\cdots$ \\
\hline
\end{tabular}

Notes.

${ }^{\text {a }}$ Column density times $10^{-8}$ mole $\mathrm{cm}^{-2}$.

${ }^{\mathrm{b}}$ See the text for the discussion of calculations.

At present, the best available calculations of the mid-infrared vibrational frequencies of naphthalene dimers that we are aware of are those of Saeki et al. (2006). Using the GAUSSIAN 03 program at the MP2/cc-pVDZ level, they found four dimer structures in the local minimum. Three of the dimer structures are those in which the naphthalene molecules are stacked, maximizing $\pi-\pi$ orbital overlap. These were the most stable. Depending on the precise level of theory, these were found to have binding energies of 5-10 kcal mol ${ }^{-1}$-energies comparable to the hydrogen bonds in water ice. A T-shaped dimer structure was also computed. This has a considerably weaker binding energy which lies between 3.5 and $6 \mathrm{kcal} \mathrm{mol}^{-1}$. The intramolecular frequencies of the naphthalene molecules comprising the four dimer structures all show qualitatively similar shifts relative to the IR absorption bands. Another motivation for this paper is to provide experimental data to refine these quantum chemical calculations.

\section{EXPERIMENT}

The infrared spectra presented here were obtained using the matrix-isolation technique in which a guest molecular species is trapped in a dilute solid solution of an IR-inactive host matrix. Here, varying concentrations of naphthalene were trapped within an argon matrix condensed upon an IR-transparent window for transmittance absorption spectroscopy.

The substrate is a $0.25 \mathrm{~cm}$ thick, 1 inch diameter cesium iodide window mounted on the cold head of a Sumitomo model RDK-408D cryostat with closed-cycle helium refrigerator. In these experiments, the cesium iodide window was maintained at $5 \mathrm{~K}$ throughout. The disk temperature was measured by a gold/ chromel thermocouple attached to the oxygen-free high thermal conductivity (OFHC) copper mount of the disk. The cold head is normally positioned to place the CsI window perpendicular to the infrared beam of a Digilab model TFS-4000 Fouriertransform infrared (FTIR) spectrometer for infrared absorption measurements. The cold head can be rotated $90^{\circ}$ to place the CsI window facing the argon and naphthalene deposition port.

The naphthalene sample consisted of roughly $5 \mathrm{~g}$ of Aldrich $99 \%+$ scintillation grade naphthalene kept at room temperature and dried by exposure to vacuum. The evaporating naphthalene was flowed with Matheson ultra-high purity grade argon supplied from a separate cylinder through $1 / 4$ inch stainless steel tubing. The mixture was introduced into the vacuum system through an Exair model 1010SS cylindrical nozzle aimed at the CsI surface at roughly $45^{\circ}$ to the surface normal.

Individual spectra were obtained as follows. An absorption spectrum of the $5 \mathrm{~K}$ window prior to deposition serves as the reference spectrum. The CsI window is then rotated to face the gas deposition port for codeposition of naphthalene and argon. After the matrix deposition is completed, the CsI window is rotated to its spectroscopic position and a post-deposition absorption spectrum is measured. The gas flowing from the nozzle, once established, was not altered during an experiment in order to better produce a uniform deposit. Spectra were obtained by co-adding 512 scans with an FTIR resolution of $0.5 \mathrm{~cm}^{-1}$.

The argon-to-naphthalene (denoted Ar/Nap hereafter) ratio was determined as follows. The argon deposit thickness $d$ was estimated from the interference fringes observed in the infrared absorption spectra using

$$
d=\frac{m}{n v},
$$

where $v$ is the frequency of a maximum or minimum in the interference fringes and $m$ is an integer for a maximum and a half-integer for a minimum. Roux et al. (1980) determined the refractive index $n$ of thin solid argon films at $20 \mathrm{~K}$ to be 1.225 between 700 and $3700 \mathrm{~cm}^{-1}$. This value was adopted for the argon thickness measurements despite a slight change in the argon ice density at the $5 \mathrm{~K}$ temperature of these experiments. The column density of argon is calculated assuming an argon density of $1.77 \mathrm{~g} \mathrm{~cm}^{-3}$ at $5 \mathrm{~K}$ (Dobbs and Jones 1957).

The column density of naphthalene in the argon matrix was estimated using the equation

$$
N=\frac{\int \tau d \nu}{A},
$$

where $\tau$ is the spectral absorbance of an observed band, $v$ is the frequency, and $A$ is the integrated absorbance value calculated for the analogous band in fully isolated naphthalene published by Hudgins et al. (1994). The average column density derived from all bands below $\sim 1500 \mathrm{~cm}^{-1}$ is reported here. Results of the argon matrix thickness and naphthalene concentration calculations are summarized in Table 1 .

\section{RESULTS AND DISCUSSION}

Figures 1-3 show the vibration-specific regions of the midinfrared spectra of matrices with $\mathrm{Ar} / \mathrm{Nap}$ ratios of approximately 1000, 530, 200,160,47, and 6 along with the spectrum of pure naphthalene measured using the same apparatus. The spectra are presented on normalized scales to highlight the spectral changes that occur as the naphthalene dilution of the samples is increased from highly isolated $(\mathrm{Ar} / \mathrm{Nap}=1000)$ to highly aggregated (Ar/Nap $=6$ and pure naphthalene). The positions and full widths at half-height (FWHH) of the unscaled absorption bands are listed in Table 2 along with peak positions for naphthalene theoretically predicted using Density functional theory (DFT). Throughout these discussions, band positions will be rounded off to the nearest wavenumber and FWHH to $0.1 \mathrm{~cm}^{-1}$. Peak positions are given in Table 2 to $0.1 \mathrm{~cm}^{-1}$.

Perusal of Figures 1-3 and the band positions in Table 2 show that spectral changes are induced by naphthalene clustering. The extent of band shift, band broadening, and effect on cross 


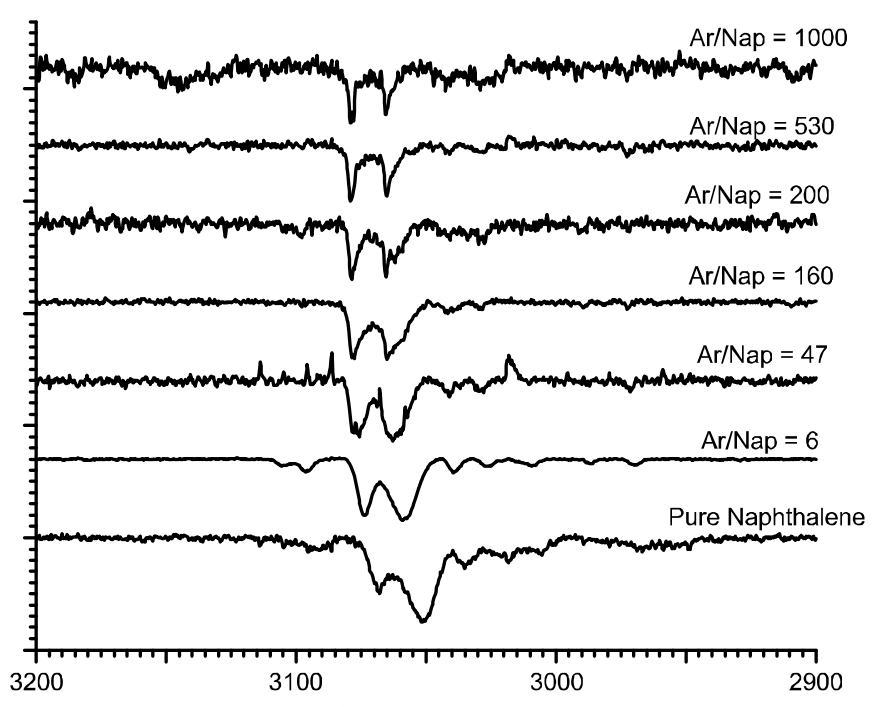

Figure 1. The 3200-2900 $\mathrm{cm}^{-1}$ absorbance spectra of argon/naphthalene matrices (Ar/naphthalene $\sim 1000,530,200,160,47$, and 6) and the $3200-2900 \mathrm{~cm}^{-1}$ spectrum of pure naphthalene. Traces are normalized so that the higher frequency $b_{2 u}$ band (see the text) has unit height and are vertically shifted for clarity.

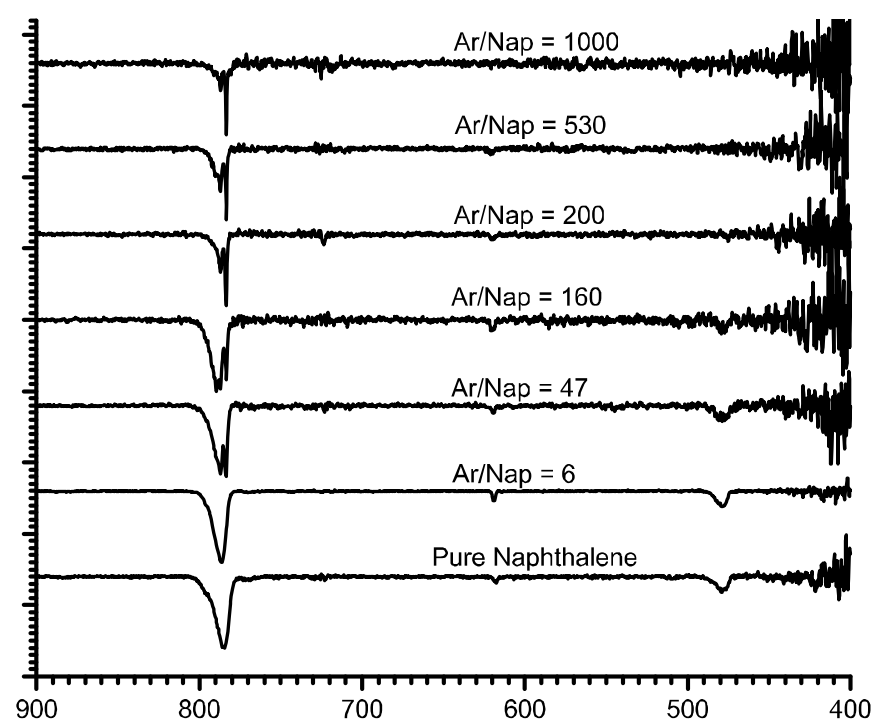

Figure 2. The $900-400 \mathrm{~cm}^{-1}$ absorbance spectra of argon/naphthalene matrices (Ar/naphthalene $\sim 1000,530,200,160,47$, and 6) and the 900-400 $\mathrm{cm}^{-1}$ spectrum of pure naphthalene. Traces are normalized so that the strongest component of the $\mathrm{CH}$ in-plane bending complex at $\sim 783 \mathrm{~cm}^{-1}$ has unit height and are vertically shifted for clarity.

section depend somewhat on the molecular vibration involved. Both the in-plane and out-of-plane pure $\mathrm{C}-\mathrm{H}$ vibrational modes are more strongly influenced than those involving pure $\mathrm{C}-\mathrm{C}$ or mixed $\mathrm{C}-\mathrm{C}$ and $\mathrm{C}-\mathrm{H}$ vibrations. Consequently, the results are presented in several subsections based on the nature of the affected vibrations.

The fraction of naphthalene molecules existing as isolated molecules within the argon matrix as a function of the naphthalene volume fraction was estimated using a computer model based upon the algorithm of Månsson \& Rudemo (2002). Each naphthalene molecule is modeled as a three-dimensional, oblate, hard spherocylinder (or OHSC), which is the set of points that are at a distance $R$ from a two-dimensional, planar disk of radius $\sigma$ (Cuetos and Martínez-Haya 2008). The van der Waals dimensions of a naphthalene molecule are given as $8.12 \AA \times 7.36 \AA$

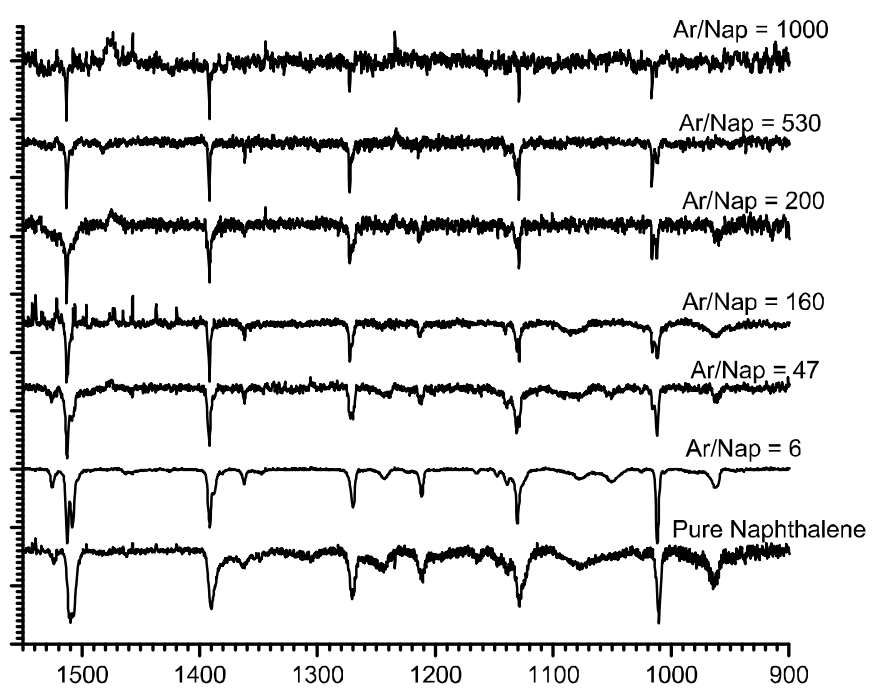

Figure 3. The $1550-900 \mathrm{~cm}^{-1}$ absorbance spectra of argon/naphthalene matrices (Ar/naphthalene $\sim 1000,530,200,160,47$, and 6) and the $1550-900 \mathrm{~cm}^{-1}$ spectrum of pure naphthalene. Traces are normalized so that the peak at $\sim 1392 \mathrm{~cm}^{-1}$ has unit height and are vertically shifted for clarity.

$\times 3.4 \AA$ in Hallmark et al. (1991). We choose $R=1.7 \AA$ and $\sigma=3.23 \AA$, corresponding to an OHSC thickness of $2 R=3.4 \AA$ and outer radius $R+\sigma=4.93 \AA$ (the mean caliper radius of an $8.12 \AA \times 7.36 \AA$ rectangle), to reproduce the van der Waals' thickness and mean radius of a naphthalene molecule.

The steps of the computation for each simulation are as follows. First, a user-input intensity parameter for a Poisson distribution was used to generate the random number of trial OHSCs within a $200 \AA$ cube. The center of each OHSC was randomly positioned within this cube and given a random unit vector for its orientation in space. Probability distributions for the positions and orientations were uniformly distributed over the full cube and the full set of unit vectors respectively with independent values given to each OHSC.

Second, the global thinning procedure of Månsson \& Rudemo (2002) was carried out. Each trial OHSC is given a random, independent mark from a uniform probability distribution over the range $0-1$. If any two OHSCs are determined to be intersecting, the one with the higher mark is retained and the other is deleted. Both OHSCs are deleted if their marks are equal. Distances between OHSCs were computed using the iterative procedure described by Cuetos and Martínez-Haya (2008) to a tolerance of $10^{-4} \AA$.

To evade edge effects, only the surviving OHSCs within the central $140 \AA$ cube are used to compute statistics. The volume fraction generated by a set of 1000 simulations is given by the average number of the surviving OHSCs per unit volume inside of this central cube times the OHSC volume. The monomer fraction of a set is taken to be the total fraction of these survivors of that set that had no nearest neighbor closer than $3.76 \AA$ (the diameter of an argon atom). The volume fraction and the monomer fraction were calculated for 10 sets of 1000 simulations in order to calculate a mean value and a standard error of the mean. These values were determined for a range of initial intensity parameters between $10^{-6}$ and $10^{-3} \AA^{-3}$.

The monomer fraction as a function of 1 minus the volume fraction for this set of initial intensity parameters is shown as the filled squares in the log-log plot in Figure 4. These points are well represented by a power law with power $26.3 \pm 0.5$. Since each naphthalene molecule replaces approximately four 
Table 2

Experimentally Measured Naphthalene Band Positions (FWHH in Parentheses) Compared to the Theoretically Computed Spectrum Using DFT

\begin{tabular}{|c|c|c|c|c|c|c|}
\hline Ar/Nap $=1000$ & $\mathrm{Ar} / \mathrm{Nap}=530$ & $\mathrm{Ar} / \mathrm{Nap}=200$ & $\mathrm{Ar} / \mathrm{Nap}=160$ & Ar/Nap $=47$ & $\mathrm{Ar} / \mathrm{Nap}=6$ & DFT $^{\mathrm{a}}$ \\
\hline \multicolumn{7}{|c|}{$\mathrm{C}-\mathrm{C}-\mathrm{C}$ and $\mathrm{C}-\mathrm{H}$ out-of-plane bending region } \\
\hline$\ldots$ & & $475.2(1.7)$ & $479.1(6.2)$ & $478.8(8.9)$ & $478.6(7.5)$ & 480.0 \\
\hline$\ldots$ & $621.1(4.0)$ & $619.6(2.2)$ & $620.1(3.2)$ & $619.6(2.2)$ & $618.9(1.9)$ & $\ldots$ \\
\hline$\ldots$ & $624.2(0.5)$ & $620.8(1.0)$ & 620.8 & $\ldots$ & $\ldots$ & 632.1 \\
\hline $783.6(0.9)$ & $783.6(0.9)$ & $783.6(1.3)$ & $783.6(1.7)$ & $783.6(9.2)$ & 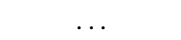 & 788.4 \\
\hline $787.2(2.6)$ & $787.2(5.1)$ & $787.2(3.0)$ & $787.2(7.7)$ & 787.2 & $786.2(8.5)$ & $\ldots$ \\
\hline$\ldots$ & $\ldots$ & $\ldots$ & 789.3 & $\ldots$ & $\ldots$ & $\ldots$ \\
\hline \multicolumn{7}{|c|}{$\mathrm{C}-\mathrm{C}$ stretching and $\mathrm{C}-\mathrm{H}$ in-plane bending region } \\
\hline ... & $\ldots$ & $960.1(6.8)$ & $961.8(11.2)$ & $961.3(3.8)$ & $962.5(8.0)$ & $\ldots$ \\
\hline$\ldots$ & $\ldots$ & 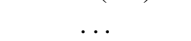 & & $963.4(2.5)$ & & 963.5 \\
\hline $1012.6(1.4)$ & $1011.7(2.6)$ & $1012.1(1.4)$ & $1012.1(4.2)^{\mathrm{b}}$ & $1012.1(2.7)$ & $1011.9(2.9)$ & 1009.7 \\
\hline $1016.5(1.2)$ & $1016.5(1.4)$ & $1016.2(1.4)$ & $1016.0(2.2)^{b}$ & 1016.0 & $\ldots$ & $\ldots$ \\
\hline$\ldots$ & $\ldots$ & $\ldots$ & & $1051.2(7.4)$ & $1051.0(12.2)$ & $\ldots$ \\
\hline$\ldots$ & $\ldots$ & $\ldots$ & $1085.2(19.6)$ & $1078.7(26.2)$ & $1077.5(13.4)$ & $\ldots$ \\
\hline $1129.3(0.7)$ & $1129.3(1.0)$ & $1129.3(0.1)$ & $1129.1(1.4)$ & $1129.3(2.7)^{b}$ & $1130.5(3.5)$ & $\ldots$ \\
\hline $1131.0(1.0)$ & $1131.0(3.0)^{\mathrm{b}}$ & $1131.0(3.3)^{\mathrm{b}}$ & $1131.3(2.3)^{b}$ & $1131.3(3.2)^{\mathrm{b}}$ & $\ldots$ & 1131.9 \\
\hline$\ldots$ & $\ldots$ & $\ldots$ & $1140.7(2.2)$ & $1139.5(5.9)$ & $1139.5(5.0)^{\mathrm{b}}$ & $\ldots$ \\
\hline$\ldots$ & $\ldots$ & $\ldots$ & $\ldots$ & $\ldots$ & $1148.1(3.9)$ & $\ldots$ \\
\hline$\ldots$ & $\ldots$ & $\ldots$ & $\ldots$ & $1161.6(0.6)$ & $1165.3(4.1)$ & 1169.7 \\
\hline$\cdots$ & $\ldots$ & $1212.5(1.2)^{\mathrm{c}}$ & 1212.5 & $1212.0(2.4)^{b}$ & $1211.8(3.5)$ & 1208.8 \\
\hline$\ldots$ & $1214.4(0.5)$ & $1214.0(1.7)$ & $1213.7(4.1)$ & $1213.7(2.6)^{\mathrm{b}}$ & $\ldots$ & $\ldots$ \\
\hline$\ldots$ & $\ldots$ & $\ldots$ & $1245.5(1.0)$ & $1243.8(13.2)$ & $1243.6(7.8)$ & $\ldots$ \\
\hline $1273.0(0.9)$ & $1272.8(1.4)$ & $1272.8(4.2)$ & $1272.8(3.3)$ & $1270.1(5.0)$ & $1270.1(4.4)$ & 1270.0 \\
\hline$\ldots$ & $\ldots$ & $\ldots$ & $\ldots$ & $1345.8(1.3)$ & $\ldots$ & $\ldots$ \\
\hline$\ldots$ & $\ldots$ & $\ldots$ & $\ldots$ & 1346.6 & $1347.8(9.1)$ & $\ldots$ \\
\hline$\ldots$ & $1362.0(1.0)$ & $1362.2(2.0)$ & $1362.0(1.7)$ & $1362.0(2.3)$ & $1362.7(3.1)$ & 1358.3 \\
\hline $1392.1(1.0)$ & $1392.1(1.0)$ & $1391.9(1.9)$ & $1391.9(1.7)$ & $1391.9(2.6)$ & $1391.6(3.2)$ & 1399.8 \\
\hline$\ldots$ & & $\ldots$ & & 1508.6 & $1508.1(4.3)^{\mathrm{b}}$ & 1507.9 \\
\hline $1513.2(0.9)$ & $1513.2(1.5)$ & $1512.9(1.7)$ & $1512.9(2.4)$ & $1512.4(2.2)$ & $1512.4(2.9)$ & $\ldots$ \\
\hline$\ldots$ & $\ldots$ & $\ldots$ & $\ldots$ & $1525.9(9.4)$ & $1525.5(3.6)$ & $\ldots$ \\
\hline \multicolumn{7}{|c|}{$\mathrm{C}-\mathrm{H}$ stretching region } \\
\hline$\ldots$ & $\ldots$ & $\ldots$ & $\ldots$ & $\ldots$ & $2926.7(5.1)$ & $\ldots$ \\
\hline$\cdots$ & $\ldots$ & $\ldots$ & $\ldots$ & $\cdots$ & $2986.4(3.8)$ & $\cdots$ \\
\hline$\ldots$ & $\ldots$ & $\ldots$ & $\ldots$ & $\ldots$ & $3009.4(6.5)$ & $\ldots$ \\
\hline $3029.2(15.2)^{\mathrm{b}}$ & $3027.8(5.4)$ & $3029.7(18.8)^{\mathrm{d}}$ & $3028.7(3.7)$ & $3027.8(4.9)$ & $3026.6(5.3)$ & $\ldots$ \\
\hline $3042.5(12.9)^{\mathrm{c}}$ & $3041.0(3.3)$ & 3040.8 & $3041.5(6.7)$ & $3041.0(4.0)$ & $3039.6(5.0)$ & 3043.5 \\
\hline$\ldots$ & $\ldots$ & $\ldots$ & $\ldots$ & $\ldots$ & $\ldots$ & 3045.4 \\
\hline$\ldots$ & $\ldots$ & 3062.0 & 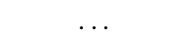 & $\ldots$ & $\ldots$ & $\ldots$ \\
\hline $3065.6(3.8)$ & $3065.1(4.1)$ & $3065.4(7.6)$ & $3064.9(10.9)$ & $3063.0(10.4)$ & $3059.1(12.6)$ & 3062.5 \\
\hline $3078.9(2.9)$ & $3079.1(3.2)$ & $3078.4(4.0)$ & $3077.7(7.3)$ & $3075.7(7.1)$ & $3073.8(7.2)$ & 3076.5 \\
\hline$\ldots$ & $\ldots$ & $\ldots$ & $\ldots$ & $\ldots$ & $3096.5(5.7)$ & $\ldots$ \\
\hline$\ldots$ & $\ldots$ & $\ldots$ & $\ldots$ & $\ldots$ & $3105.4(6.9)^{\mathrm{b}}$ & $\ldots$ \\
\hline
\end{tabular}

Notes. Units of $\mathrm{cm}^{-1}$ for all entries.

${ }^{\text {a }}$ DFT calculations from Langhoff (1996).

b Peak is only partially resolved. FWHH measured up to interpeak minimum.

${ }^{\mathrm{c}}$ Peak is just barely resolved. FWHH measured up to interpeak minimum.

${ }^{\mathrm{d}}$ Quoted FWHH is for entire complex of partially resolved peaks.

argon atoms within the matrix (Crépin et al. 2001), we use the naphthalene/argon ratio times four as the volume fraction for the purpose of estimating the monomer fraction within our experimental data. The monomer fraction calculated using this power law for the experimental results is listed in Table 1 and is shown as the open circles in Figure 4.

\subsection{The C-H Stretching Region (3200-2900 $\left.\mathrm{cm}^{-1}\right)$}

Figure 1 shows the $3200-2900 \mathrm{~cm}^{-1}$ region of the spectrum of naphthalene in argon matrices at different concentrations and that of pure naphthalene. This region of the spectrum is dominated by two strong bands, both assignable to $\mathrm{C}-\mathrm{H}$ stretching vibrations based on good agreement with the frequencies predicted by DFT calculations using the 4-31G expansion basis set and B3LYP functional (Langhoff 1996). The spectral changes in going from the top to the bottom spectrum are caused by increasing interactions between naphthalene molecules as their concentration increases. These interactions perturb the vibrational force field, inducing changes in fundamental vibrational frequencies.

The $3079 \mathrm{~cm}^{-1}$ band in the $\mathrm{Ar} / \mathrm{Nap}=1000$ spectrum corresponds to a vibration with $b_{2 u}$ symmetry. With increasing fractional concentration of naphthalene, the $b_{2 u} \mathrm{C}-\mathrm{H}$ stretching mode broadens and is redshifted to $3068 \mathrm{~cm}^{-1}$ in pure naphthalene. The first five spectra in Figure 1 show that this $11 \mathrm{~cm}^{-1}$ shift 


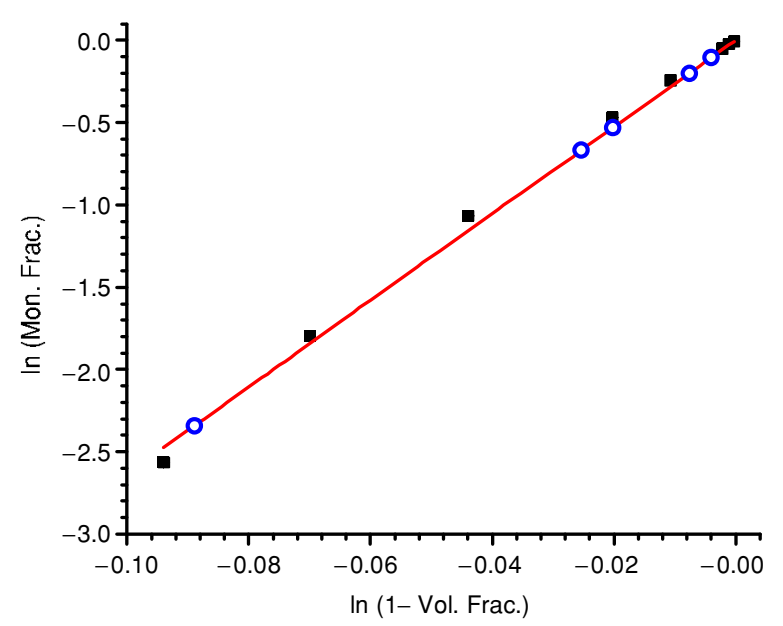

Figure 4. Black squares are the simulated monomer fraction as a function of 1 minus the volume fraction for initial intensities of $10^{-6}, 5 \times 10^{-6}, 10^{-5}$ $5 \times 10^{-5}, 10^{-4}, 2.5 \times 10^{-4}, 5 \times 10^{-4}$, and $10^{-3} \AA^{-3}$. Statistical error bars are the size of the squares. The solid line is an unweighted linear fit to the data points constrained to pass through the origin and the fitted slope is $26.3 \pm 0.5$. The open circles are the monomer fractions calculated for the experimental results using this power law (see the text).

(A color version of this figure is available in the online journal.)

is not gradual, but rather caused by the growth of perhaps two distinct new features which produce a red shoulder that grows as the Ar/Nap concentration decreases. These bands, centered near $3075 \mathrm{~cm}^{-1}$, overlap and ultimately overtake the narrower $3079 \mathrm{~cm}^{-1}$ matrix-isolated naphthalene band, which appears to be completely replaced when $\mathrm{Ar} / \mathrm{Nap}=6$. It is significant that the peak position of the $b_{2 u} \mathrm{C}-\mathrm{H}$ stretching band produced by naphthalene agglomerations at Ar/Nap $=6\left(3074 \mathrm{~cm}^{-1}\right)$ is different from that of pure naphthalene $\left(3068 \mathrm{~cm}^{-1}\right)$ as shown in the bottom spectrum. This indicates that the structure of the naphthalene agglomeration produced at very high concentrations and the corresponding intermolecular interactions are different from those in pure naphthalene.

The $3066 \mathrm{~cm}^{-1}$ band in the Ar/Nap = 1000 spectrum corresponds to a vibration with $b_{1 u}$ symmetry. The $b_{1 u} \mathrm{C}-\mathrm{H}$ stretching mode shows similar behavior to its nearby partner. The shoulder that grows with increasing concentration of naphthalene peaks at about $3062 \mathrm{~cm}^{-1}$, some $4 \mathrm{~cm}^{-1}$ to the red of the original, and the same as the $4 \mathrm{~cm}^{-1}$ offset of the red shoulder growing adjacent to the $3079 \mathrm{~cm}^{-1} b_{2 u}$ band. However, the $15 \mathrm{~cm}^{-1}$ redshift between the $b_{1 u}$ vibration for the highly dilute spectrum versus that for pure naphthalene is some $5 \mathrm{~cm}^{-1}$ greater than the $10 \mathrm{~cm}^{-1}$ redshift for the $3079 \mathrm{~cm}^{-1}$ $b_{2 u}$ vibration.

The effect of increasing naphthalene concentration in the argon matrix can produce spectral shifts as follows. In the most dilute case (Ar/Nap $=1000$, adjusted to 250 for this calculation), the fraction of naphthalene molecules that are isolated in the matrix is $0.90 \pm 0.03$. Consequently, since the infrared absorption bands arise primarily from isolated naphthalene molecules, the bands in the Ar/Nap $=1000$ spectrum are well defined and have widths on the order of a few $\mathrm{cm}^{-1}$. At $\mathrm{Ar} / \mathrm{Nap}=530$, the monomer fraction drops slightly $(0.82 \pm 0.03)$, consistent with little or no change in the spectrum. The Ar/Nap $=200$ spectrum shows a strong red wing on both features. In this case, the monomer fraction has dropped to $0.59 \pm 0.08$. At $\mathrm{Ar} / \mathrm{Nap}=160$, this fraction becomes $0.51 \pm 0.09$, consistent with larger red shoulders.
At $\mathrm{Ar} / \mathrm{Nap}=47$, the monomer fraction is only $0.10 \pm 0.04$. Not surprisingly, it is at this concentration that the new bands producing the shoulders overtake the initial narrow features. At $\mathrm{Ar} / \mathrm{Nap}=6$, our equation for the monomer fraction breaks down, since the volume fraction of naphthalene exceeds the maximal limit of 0.125 for the Månsson \& Rudemo (2002) global thinning process. Ar/Nap $=6$ clearly corresponds to massive agglomeration of naphthalene molecules within the matrix with a significant change in the spectrum in going from $\mathrm{Ar} / \mathrm{Nap}=47$ to $\mathrm{Ar} / \mathrm{Nap}=6$. There is no longer a contribution at the initial frequencies, but now both bands are symmetric with Gaussian profiles. There still remains a significant difference between the spectra of these agglomerations and that of pure naphthalene as shown by comparing the bottom two spectra in Figure 1.

\subsection{The C-H Out-of-plane Bending Region (900-400 $\mathrm{cm}^{-1}$ )}

Figure 2 shows the $900-400 \mathrm{~cm}^{-1}$ region of the argon matrixisolated spectra discussed above along with the corresponding spectrum of pure naphthalene. For aromatic molecules, the strong bands in this region originate in $\mathrm{C}-\mathrm{H}$ out-of-plane $\left(\mathrm{CH}_{\text {oop }}\right)$ bending vibrations and they are characteristic of the number of adjacent hydrogen atoms on each ring (e.g., Silverstein \& Bassler 1967; Bellamy 1960). A feature near $790 \mathrm{~cm}^{-1}$ dominates this region for naphthalene because it has only four adjacent (quartet) hydrogens per ring (e.g., Hudgins \& Allamandola 1999). This is readily assigned to the $b_{3 u} \mathrm{CH}_{\text {oop }}$ bend, again based on good agreement with the frequency $788.4 \mathrm{~cm}^{-1}$ predicted by DFT calculations (Langhoff 1996). As with the $\mathrm{C}-\mathrm{H}$ stretching bands discussed above, the spectral changes in going from the top to the bottom spectrum are caused by increasing interactions between naphthalene molecules as their concentration increases. However, for the $\mathrm{CH}_{\text {oop }}$ bending mode, the band shift upon clustering is to the blue not the red as is the case for the $\mathrm{C}-\mathrm{H}$ stretching modes.

For the most dilute cases (Ar/Nap $=1000,530$, and 200), a strong, narrow band (784 $\mathrm{cm}^{-1}$, FWHM $\left.0.9 \mathrm{~cm}^{-1}\right)$ accompanied by one or more well-defined satellite bands dominates the entire region covered in Figure 2. The $784 \mathrm{~cm}^{-1}$ band is readily assigned to isolated naphthalene and falls within $2 \mathrm{~cm}^{-1}$ of the position measured for gas-phase naphthalene near room temperature (Pirali et al. 2009). For the $\mathrm{CH}_{\text {oop }}$ bending feature, the overlapping satellite bands overtake the isolated naphthalene $784 \mathrm{~cm}^{-1}$ band at Ar/Nap $=160$. This is not the case for the $\mathrm{C}-\mathrm{H}$ stretching features until $\mathrm{Ar} / \mathrm{Nap}=47$ (Figure 1). As with the band profile, width and position in the $\mathrm{CH}$ stretching region, the spectrum changes significantly in going from $\mathrm{Ar} / \mathrm{Nap}=47$ to $\mathrm{Ar} / \mathrm{Nap}=6$. The narrow band and broader blue satellite feature are now replaced by a strong, Gaussianlike band peaking at $786 \mathrm{~cm}^{-1}$ arising from agglomerations of naphthalene molecules. Interestingly, the peak position of this broad band is not significantly shifted from the central position of the narrow band and satellite features associated with the more dilute samples nor is there much difference with the band produced by pure naphthalene which peaks at $785 \mathrm{~cm}^{-1}$.

Two other bands fall in this spectral window, one near $620 \mathrm{~cm}^{-1}$ and the other near $479 \mathrm{~cm}^{-1}$. Again, for the most dilute mixtures, these bands fall within $2 \mathrm{~cm}^{-1}$ of those for gas-phase naphthalene (Pirali et al. 2009). Consistent with their origin in $\mathrm{C}-\mathrm{C}-\mathrm{C}$ skeletal vibrations, both are quite weak. Apart from slight broadening and at most a few $\mathrm{cm}^{-1}$ shift, both of these bands show little change with increasing naphthalene concentration. 


\subsection{The $C-C$ Stretching and $C-H$ In-plane Bending Region $\left(1550-900 \mathrm{~cm}^{-1}\right)$}

Figure 3 shows the $1550-900 \mathrm{~cm}^{-1}$ region of these matrixisolated naphthalene spectra, a region rich in bands. For aromatic molecules, features between roughly 1700 and $1500 \mathrm{~cm}^{-1}$ arise from $\mathrm{C}-\mathrm{C}$ stretching vibrations, those in the $1500-1250 \mathrm{~cm}^{-1}$ region are associated with vibrations in which $\mathrm{C}-\mathrm{C}$ stretching and $\mathrm{C}-\mathrm{H}$ in-plane $\left(\mathrm{CH}_{\mathrm{ip}}\right)$ bending motions are coupled, while those between 1250 and $1100 \mathrm{~cm}^{-1}$ primarily arise from uncoupled $\mathrm{CH}_{\mathrm{ip}}$ bending vibrations. The nature of these vibrations is again readily assigned by comparison with the frequencies predicted by DFT calculations (Langhoff 1996). The peak frequencies for the principle bands we measure for matrix-isolated naphthalene all lie within $5 \mathrm{~cm}^{-1}$ of their corresponding gasphase counterparts as reported by Pirali et al. (2009). As above, the changes in going from the top to the bottom spectrum are due to increasing interactions between naphthalene molecules as their concentration increases.

The discussions of the overall changes in the $\mathrm{C}-\mathrm{H}$ stretching and bending regions with increasing naphthalene concentration discussed above can be applied to most of the changes shown in Figure 3. The bands are sharp and narrow in the most dilute case and broaden as satellite features grow in as the naphthalene concentration increases. With one exception, all of the naphthalene cluster bands fall, at most, a few wavenumbers to the red of the band associated with matrix-isolated naphthalene. While the bands broaden upon clustering, Table 2 shows that, for the majority of these features, the bandwidths are consistently smaller than those for the $\mathrm{C}-\mathrm{H}$ stretching and $\mathrm{CH}_{\text {oop }}$ bands. The entries in Table 2 also show that the band shifts for most of the features in this region are modest, on the order of 1 or $2 \mathrm{~cm}^{-1}$, similar to the shifts that occur with the $\mathrm{CH}_{\text {oop }}$ modes. Taken together with the knowledge that most of the bands in this region arise from $\mathrm{C}-\mathrm{C}$ stretching vibrations coupled with $\mathrm{CH}_{\mathrm{ip}}$ bending vibrations, the narrow bands and small shifts for most of the features in this region are a clear indication that clustering does not significantly influence the bonding within the naphthalene carbon skeleton nor does it influence the amplitudes of the different carbon-carbon vibrations that produce these bands.

There is however a portion of this region of the spectrum which does show unusual changes as clustering increases. A narrow band (FWHM $<1 \mathrm{~cm}^{-1}$ ) at $1131 \mathrm{~cm}^{-1}$ is the only feature present between 1140 and $1100 \mathrm{~cm}^{-1}$ in the spectrum of the $\mathrm{Ar} / \mathrm{Nap}=1000$ sample. A blue wing grows in the $\mathrm{Ar} / \mathrm{Nap}=530$ and 200 matrices. This starts to change considerably at $\mathrm{Ar} / \mathrm{Nap}=160$. By $\mathrm{Ar} / \mathrm{Nap}=47$, a red shoulder is clearly present, giving the impression that the sharp $1131 \mathrm{~cm}^{-1}$ and $1139 \mathrm{~cm}^{-1}$ bands are now atop a broad underlying component. This behavior continues through the Ar/Nap $=6$ sample, where the broad component spans the $1150-1120 \mathrm{~cm}^{-1}$ range in a manner similar to that in the spectrum of pure naphthalene. Recall that these bands fall squarely in the $\mathrm{CH}_{\mathrm{ip}}$ bending region. Thus, the greatest changes in the $1550-900 \mathrm{~cm}^{-1}$ region can be understood as arising from the stronger effect naphthalene clustering has on the peripheral $\mathrm{C}-\mathrm{H}$ bonds than on the skeletal $\mathrm{C}-\mathrm{C}$ bonds.

\section{ASTROPHYSICAL IMPLICATIONS}

It is important to keep in mind that this study is limited to clusters of neutral naphthalene, a very small aromatic molecule by interstellar and solar system standards, and care is warranted in applying these results to observational data. However, in view of the paucity of information on the spectroscopic effects that clustering has on PAH infrared spectra, a few interesting points emerge which merit some discussion in an astrophysical context.

The CH stretching region. Since emission in the $\mathrm{CH}$ stretching region is dominated by the smallest members of the astronomical PAH population (Schutte et al. 1993), small PAH clusters containing on the order of 30-50 carbon atoms may contribute to the emission in this region.

The $\mathrm{CH}_{i p}$ bending region. A broad plateau that underlies the strong $7.7 \mu \mathrm{m}$ emission band complex (Cohen et al. 1986, 1989) shows different spatial behavior than that of the narrower PAH emission features (Bregman et al. 1989). Rapacioli et al. (2005) carried out a detailed study of the spatial behavior of the emission in this region in two extended objects, NGC 7023 and $\rho$ Oph-SR3. Focusing on the region near $7.7 \mu \mathrm{m}$, they analyze the emission in terms of three components, neutral PAHs, PAH cations, and Very Small Grains (VSGs). They propose that PAH molecules are trapped in VSGs, perhaps as PAH clusters.

In view of this, it is interesting that the strongest effect clustering has on the $1500-900 \mathrm{~cm}^{-1}(667-11 \mu \mathrm{m})$ region in the spectrum of naphthalene is an overall broadening of the features with red and blue wings stretching from 1150 to $1100 \mathrm{~cm}^{-1}(8.7-9 \mu \mathrm{m})$, the region of the $\mathrm{CH}_{\mathrm{ip}}$ vibrations for quartet hydrogens. Quartet hydrogens are far less important than solo hydrogens in the astronomical PAH population. If this observed strong broadening for naphthalene clusters generalizes to PAH clusters with primarily solo hydrogens, one could speculate that clustering of interstellar PAHs might in general produce a series of broad features near the $7.7 \mu \mathrm{m}$ interstellar band since that is where the $\mathrm{CH}_{\mathrm{ip}}$ bending vibrations of solo hydrogens are most strong.

The $\mathrm{CH}_{\text {oop }}$ bending region. Two of the puzzles remaining in deciphering the astronomical spectrum in this region are understanding the peculiar blue wing of the astronomical $787 \mathrm{~cm}^{-1}(12.7 \mu \mathrm{m})$ band (Hony et al. 2001; Bauschlicher et al. 2008) and the very broad plateau which underlies the entire region between roughly 900 and $700 \mathrm{~cm}^{-1}(11-14.3 \mu \mathrm{m})$ (Roche et al. 1989).

The $12.7 \mu \mathrm{m}$ astronomical band is attributed to the $\mathrm{CH}_{\text {oop }}$ bending vibration of astronomical PAHs with duo and trio hydrogens (see discussion in Bauschlicher et al. (2008), and references therein). However, whereas redshifting and band broadening are not unusual, blue shading is enigmatic among the astronomical PAH emission features. The observed blue shading of the naphthalene $\mathrm{CH}_{\text {oop }}$ band to $786 \mathrm{~cm}^{-1}(12.7 \mu \mathrm{m})$ with decreasing Ar/Nap ratio is temptingly consistent with the phenomenon.

If PAH clusters in general contribute to the blue component, one would predict spatial variation between the main peak and the blue wing. Since there seem to be few studies of the spatial behavior of the $12.7 \mu \mathrm{m}$ feature in extended sources, the work of Roche et al. (1989) on NGC 7027 and Rapacioli et al. (2005) on NGC 7023 is particularly important. Both studies indicate that the $12.7 \mu \mathrm{m}$ band and its blue wing show different spatial behavior with position in the object, implying that these two components track different members of the interstellar PAH family. Turning to the underlying plateau, Roche et al. (1989) clearly show that it tracks a separate component of the astronomical PAH family, one which extends well beyond the region in which the PAH features are strong. This behavior is similar to that reported by Rapacioli et al. (2005) for the bands in the NGC 7023. Clearly, the spectroscopic effects observed here must be tested with further studies on clusters involving 
larger PAHs with different edge structures, mixed PAH clusters, and PAH clusters with different electrical charge, all species which likely make up the astronomical PAH population. Since PAH clusters are model components of Jupiter (Friedson et al. 2002) and Titan's atmospheres (Wilson \& Atreya 2003), the information presented here may also be applicable to the spectroscopy of these objects.

\section{CONCLUSIONS}

This paper describes the changes in the infrared spectrum of naphthalene that occur as isolated naphthalene molecules agglomerate into clusters. As naphthalene clusters grow, bands shift and broaden. The strongest perturbations are associated with $\mathrm{C}-\mathrm{H}$ vibrations, not skeletal $\mathrm{C}-\mathrm{C}$ vibrations. Spectral shifts are modest, some $4-12 \mathrm{~cm}^{-1}$, and bands are broadened to $5-10 \mathrm{~cm}^{-1}$. The possible contribution of homogeneous naphthalene clusters to the widespread interstellar infrared emission spectrum is discussed. Specifically, the likelihood that PAH clusters contribute to the emission plateau that underlies the $7.7 \mu \mathrm{m}$ and $8.6 \mu \mathrm{m}$ features and the blue shading of the $12.7 \mu \mathrm{m}$ emission band is considered.

The authors gratefully acknowledge sustained support from NASA's Laboratory Astrophysics and Astrobiology Programs. The authors thank Christiaan Boersma for helpful discussions about the computer simulations described here.

\section{REFERENCES}

Ando, N., Mitsui, M., \& Nakajima, A. 2008, J. Chem. Phys., 128, 154318

Bauschlicher, C. W., Peeters, E., Jr, \& Allamandola, L. J. 2008, ApJ, 678, 316

Bauschlicher, C. W., Peeters, E., Jr, \& Allamandola, L. J. 2009, ApJ, 697, 311

Bellamy, L. J. 1960, The Infra-red Spectra of Complex Molecules (2nd ed.; New York: Wiley)

Benharash, P., Gleason, M. J., \& Felker, P. M. 1999, J. Phys. Chem. A, 103, 1442

Boersma, C., Bauschlicher, C. W., Jr., Allamandola, L. J., Peeters, E., \& Tielens, A. G. G. M. 2010, A\&A, 511, A32

Boersma, C., Peeters, E., Martín-Hernádez, N. L., van der Wolk, G., Verhoeff, A. P., Tielens, A. G. G. M., Waters, L. B. F. M., \& Pel, J. W. 2009, A\&A, 502,175

Bregman, J. D., Allamandola, L. J., Tielens, A. G. G. M., Geballe, T. R., \& Witteborn, F. C. 1989, ApJ, 344, 791

Cohen, M., Allamandola, L. J., Tielens, A. G. G. M., Bregman, J., Simpson, J., Witteborn, F. C., Wooden, D., \& Rank, D. 1986, ApJ, 302, 737

Cohen, M., Tielens, A. G. G. M., Bregman, J. D., Witteborn, F. C., Rank, D. M., Allamandola, L. J., Wooden, D., \& de Muizon, M. 1989, ApJ, 341, 246

Crépin, C., de Pujo, P., Bouvier, B., Brenner, V., \& Millié, Ph. 2001, Chem. Phys., 272, 243

Cuetos, A., \& Martínez-Haya, B. 2008, J. Chem. Phys., 129, 214706

Dobbs, E. R., \& Jones, G. O. 1957, Rep. Prog. Phys., 20, 516

Friedson, A. J., Wong, A.-S., \& Yung, Y. L. 2002, Icarus, 158, 389

Fujiwara, T., \& Lim, E. C. 2003, J. Phys. Chem. A, 107, 4381

Gilliéron, C., Sharma, N., Nauta, K., \& Schmidt, T. W. 2007, J. Phys. Chem. A, 111,4211
Hallmark, V. M., Chiang, S., Brown, J. K., \& Wöll, Ch. 1991, Phys. Rev. Lett., 66,48

Hony, S., Van Kerckhoven, C., Peeters, E., Tielens, A. G. G. M., Hudgins, D. M., \& Allamandola, L. J. 2001, A\&A, 370, 1030

Hudgins, D. M., \& Allamandola, L. J. 1999, ApJ, 516, L41

Hudgins, D. M., Bauschlicher, C. W., Jr., \& Allamandola, L. J. 2005, ApJ, 632, 316

Hudgins, D. M., Sandford, S. A., \& Allamandola, L. J. 1994, J. Phys. Chem., 98,4243

Iglesias-Groth, S., Manchado, A., García-Hernández, D. A., Hernández, J. I. G., \& Lambert, D. L. 2008, ApJ, 685, L55

Joblin, C., Berné, O., Simon, A., \& Mulas, G. 2009, in ASP Conf. Ser. 414, Cosmic Dust-Near and Far, ed. T. Henning, E. Grün, \& J. Steinacker (San Francisco, CA: ASP), 383

Kim, W., Schaeffer, M. W., Lee, S., Chung, J. S., \& Felker, P. M. 1999, J. Chem. Phys., 110, 11264

Kimura, Y., Saito, M., Sakon, I., \& Kaito, C. 2007, in Lunar Planetary Institute Conf. 38, ed. LPI Editorial Board (Houston, TX: LPI), http://www.lpi.usra.edu/meetings/lpsc2007/pdf/1511.pdf

Knorke, H., Langer, J., Oomens, J., \& Dopfer, O. 2009, ApJ, 706, L66

Langhoff, S. R. 1996, J. Phys. Chem., 100, 2819

Månsson, M., \& Rudemo, M. 2002, Adv. Appl. Prob., 34, 718

Mattioda, A. L., Hudgins, D. M., Bauschlicher, C. W., Jr., \& Allamandola, L. J. 2005, Adv. Space Res., 36, 156

Mattioda, A. L., Hudgins, D. M., Bauschlicher, Jr., C. W., Rosi, M., \& Allamandola, L. J. 2003, J. Phys. Chem. A, 107, 1486

Mattioda, A. L., Rutter, L., Parkhill, J., Head-Gordon, M., Lee, T. J., \& Allamandola, L. J. 2008, ApJ, 680, 1243

Mulas, G., Malloci, G., Joblin, C., \& Toublanc, D. 2006, A\&A, 460, 93

Peeters, E., Allamandola, L. J., Hudgins, D. M., Hony, S., \& Tielens, A. G. G. M. 2004, in ASP Conf. Ser. 309, Astrophysics of Dust, ed. A. N. Witt, G. C. Clayton, \& B. T. Draine (San Francisco, CA: ASP), 141

Peeters, E., Mattioda, A. L., Kemper, F., Hudgins, D. M., \& Allamandola, L. J. 2006, in ASP Conf. Ser. 357, The Spitzer Space Telescope: New Views of the Cosmos, ed. L. Armus \& W. T. Reach (San Francisco, CA: ASP), 95

Pirali, O., Vervloet, M., Mulas, G., Malloci, G., \& Joblin, C. 2009, Phys. Chem. Chem. Phys., 11, 3443

Rapacioli, M., Calvo, F., Joblin, C., Parneix, P., Toublanc, D., \& Spiegelman, F. 2006, A\&A, 460, 519

Rapacioli, M., Joblin, C., \& Boissel, P. 2005, A\&A, 429, 193

Rapacioli, M., Spiegelman, F., Talbi, D., Mineva, T., Goursot, A., Heine, T., \& Seifert, G. 2009, J. Chem. Phys., 130, 344304

Ricks, A. M., Douberly, G. E., \& Duncan, M. A. 2009, ApJ, 702, 301

Roche, P. F., Aitken, D. K., \& Smith, C. H. 1989, MNRAS, 236, 485

Roux, J. A., Wood, B. E., Smith, A. M., \& Plyler, R. R. 1980, Infrared Optical Properties of Thin CO, NO, CH4, HCL, N2O, O2, N2, Ar, and Air Cryofilms (Arnold Air Force Station, TN: Arnold Engineering Development Center), Report No. AEDC-TR-79-81

Saeki, M., Akagi, H., \& Fujii, M. 2006, J. Chem. Theory Comput., 2, 1176

Saigusa, H., \& Lim, E. C. 1995, J. Phys. Chem., 99, 15738

Schaeffer, M. W., Kim, W., Maxton, P. M., Romascan, J., \& Felker, P. M. 1995, Chem. Phys. Lett., 242, 632

Schutte, W. A., Tielens, A. G. G. M., \& Allamandola, L. J. 1993, ApJ, 415, 397

Silverstein, R. M., \& Bassler, G. C. 1967, Spectrometric Identification of Organic Compounds (2nd ed.; New York: Wiley)

Tielens, A. G. G. M. 2008, ARA\&A, 46, 289

Tsuzuki, S., Honda, K., Uchimaru, T., \& Mikami, M. 2004, J. Chem. Phys., 120,647

Vijh, U. P., Witt, A. N., \& Gordon, K. D. 2005, ApJ, 619, 368

Wessel, J. E., \& Syage, J. A. 1990, J. Phys. Chem., 94, 737

Wilson, E. H., \& Atreya, S. K. 2003, Planet. Space. Sci., 51, 1017 